\title{
The prevalence and impact of oral complication in women during chemotherapy after surgery for breast cancer - A cross sectional study
}

\author{
Marlene Jensen ${ }^{1}$, Sasja Jul Haakonsen ${ }^{2}$, Preben Ulrich Pedersen*2 \\ ${ }^{1}$ Department of Oncology, Aalborg University Hospital, Hobrovej, Aalborg, Denmark \\ ${ }^{2}$ Centre of Clinical Guidelines, Department of Clinical Medicine, AAlborg University, Denmark
}

Received: July 8, 2019

DOI: $10.5430 /$ jnep.v10n2p8
Accepted: October 7, 2019

Online Published: October 22, 2019

\begin{abstract}
Background and aim: Mucositis is a well-known side effect to chemotherapy treatment after breast cancer surgery. The number of women who experience oral complication that is not classified as mucositis is less investigated as well as the impact of oral complication on the women's quality of life. Aim: To describe how many women with breast cancer report oral complications during their adjuvant chemotherapy with Ebirubicin, Cyclophosphamide and Taxotere or Taxol, to describe which oral complications the women report and the impact the oral complication has on women's daily life and quality of life.

Methods: A cross-sectional design was used. The women were invited to fill out a self-composed questionnaire at proximal 12 weeks after initiation of the treatment. The questionnaire had two scales to summarize information about oral complication and their impact on daily living was used. The questionnaire has been face- and content validated. Internal consistency was between 0.76 to 0.83 .

Results: All 101 women had experienced oral complications to some extent. A linear regression analysis has reviled that redness, coaching and changes of taste explained $74 \%$ of reported reduction in quality of life. There was a positive correlation between the sum of symptoms (number of symptoms and duration) and reported quality of life score $r=.480(p=.000)$.

Conclusions: Oral complications was experienced by all women who were treated with CT after breast cancer surgery. Redness, coaching and changes of taste were significant contributors to reducing quality of life and need to be prevented during chemotherapy.
\end{abstract}

Key Words: Mucositis, Chemotherapy treatment, Breast cancer surgery, Oral complication, Quality of life

\section{BACKGROUND}

Breast cancer is the most commonly occurring cancer in women and the second most common cancer overall. There were over 2 million new cases in 2018. ${ }^{[1]}$ Denmark is among the 25 countries with the highest rates of breast cancer in 2018. ${ }^{[1]}$
In Denmark a nationwide screening program was introduced in 2008-2009, since then an increase in the number of newly diagnosed case of breast cancer has been detected but it is expected that this increase is temporary. The primary treatment for breast cancer is surgery combined with post-treatment such as chemotherapy, radiation therapy, anti-hormonal treat-

*Correspondence: Preben Ulrich Pedersen; Email: pup@cfkr.info; Address: Centre of Clinical Guidelines, Department of Clinical Medicine, AAlborg University, Denmark. 
ment and/or treatment with the monoclonal antibody drug Trastuzmab. ${ }^{[2]}$

The number of patients for primary surgery has been relatively stable the last ten years but referrals to adjuvant chemotherapy treatment have increased significantly. The adjuvant regime with chemotherapy for the treatment of breast cancer is a combination of Epirubicin, Cyclophosphamide and Docetaxel or Taxol. ${ }^{[2,3]}$ Chemotherapy (CT) interferes with cellular metabolism of both malignant cells and normal tissues. ${ }^{[4]}$ Application of CT frequently causes severe adverse effects. Oral symptoms as mucosal lesions/mucositis; oral discomfort and pain; haemorrhage; salivary gland hypofunction and oral dryness; taste disturbances; higher susceptibility to bacterial, fungal, and viral infections; as well as changes in the oral microflora to become potentially more pathogenic has been linked to chemotherapy. ${ }^{[4]}$ Oral complication is one of the most common side effects to CT and is reported to be between $10 \%-100 \% .^{[3,5-7]}$ Studies that have reported on oral complications of patients receiving CT have included patients with a mix of cancer diagnoses such as haematological cancer, patients who is going through a stem cell transplantation, patients with head and neck cancer and patients in radiotherapy. ${ }^{[3,5-7]}$ The occurrence, severity, and duration of these oral adverse effects show a wide variability depending on underlying cancer diagnosis and disease stage in addition to different dose-intensities and combinations of chemotherapeutic drugs. ${ }^{[4]}$

Mucositis is defined as inflammatory and/or ulcerative lesions of the oral and/or gastrointestinal tract. ${ }^{[8]}$ One of the major causes of mucositis is high-dose cancer therapy. Alimentary tract mucositis refers to the expression of mucosal injury across the continuum of oral and gastrointestinal mucosa, from the mouth to the anus. From 21 studies including 2766 patients the OR for developing oral mucositis have been estimated to range from 2.29 to 13.64 with a mean OR of 4.08 in women undergoing $\mathrm{CT}$ after breast cancer surgery. ${ }^{[8]}$

Oral complications can result in dry mouth, bad breath, change of taste, reduced nutritional intake, pain, difficulty speaking and social isolation. ${ }^{[9]}$ Moreover, there is a risk that the oral complication can lead to local infections such as stomatitis and mucositis and further develop to a systemic infection. ${ }^{[10,11]}$ Women which have been diagnosed mucositis have reported changes in taste. ${ }^{[12-15]}$ Patients describe that there was great individual variation in patterns, intensity and impact of taste changes, with changes reported to have ceased in all participants within 3.5 months after treatment ended. While not all participants found reported changes "bothersome," those who did reported predominately emotional and social consequences. Taste changes were said to be influenced by, or to influence, other symptoms, for example, appetite loss, nutritional intake, early satiation, nausea, and oral problems. ${ }^{[12,13,15]}$ Daily life was impacted substantially when chemosensory and/or food-related changes were experienced and the changes influenced altered food preferences, practical constraints in daily life, and impact on social functioning. ${ }^{12]}$

Oral complications can result in increased need for medication to relive the pain. Furthermore, oral complications have been reported to negatively influence patient's quality of life through the impact on everyday life and oral complications have been rated as one of the worst side effects to CT. ${ }^{[5,9]}$ In addition, oral complications increase the costs of medication and can result in an extended hospitalization. Oral complications can lead to extended period of treatment. ${ }^{[5,6]}$

The adjuvant treatment is developed and adjusted, therefor nurses have to update their guidance of patients in order to prevent adverse effects or progression of side-effects caused by the CT. No previous study as solely reported the incidence of oral problems in women undergoing adjuvant $\mathrm{CT}$ for breast cancer. Previously published clinical guidelines have focused on prevention impact on daily living and treatment of stomatitis or mucositis during CT. It is recommended that patients assess their own mouth and report any complications, so treatment can be initiated early, and severe complications avoided and that patients understand the importance of good oral hygiene prior to and during $\mathrm{CT}$ treatments. ${ }^{[8]}$ However, from our experience more women are suffering from oral complication that those diagnosed with a stomatitis or mucositis. To provide patients with relevant education relating to oral care, and the impact on daily living more detailed information is needed on what type of oral complication the women experience, the impact on daily living and which preventive measure women initiate.

Thus, the aim of this study is to describe how many women with breast cancer report oral complications during their adjuvant CT with Ebirubicin, Cyclophosphamide and Taxotere or Taxol, to describe which oral complications the women report and the impact the oral complication has on women's daily life and quality of life.

\section{Methods}

At the oncological outpatient clinic at Aalborg university Hospital, Denmark. The department treat approximately 300 women each year with adjuvant CT. The treatment is standardized by the guideline for diagnostics, surgery, pathology and oncology set out by The Danish Breast Cancer Group (DBCG) and The national Board of Health. ${ }^{[2]}$ The $\mathrm{CT}$ is given as three treatments with Ebirubicin and $\mathrm{Cy}-$ 
clophosphamide every 3 week, followed by three treatments with Taxotere every 3 week or nine weekly treatments with Taxol. ${ }^{[2]}$

All women referred to adjuvant CT with Ebirubicin, $\mathrm{Cy}-$ clophosphamide and Taxotere or Taxol in a nine months period were consecutively included in the study. In this study, 101 women were included. Women who does not speak or read Danish, women who had a psychiatric diagnose or were diagnosed as demented were excluded.

\subsection{Design}

A cross-sectional design was used. The women were invited to fill out a self-composed questionnaire at the fifth treatment at proximal 12 weeks after initiation of the treatment. The questionnaire was filled out during treatment and returned to the staff in an envelope before the woman left the outpatient clinic.

\subsection{Instrument}

A self-composed questionnaire with two scales to summarize information about oral complication and their impact on daily living was used. The items in each scale were included after a search of the literature of reported oral cavity problems reported by patients during chemotherapy (4). Only items related to the oral cavity were included. The "Oral symptom scale" has seven items (Dry Mouth, Pain, Redness, Coating, Wound, Changes in taste, Other complications) that identify oral symptoms and the duration of the symptom. Each item could be scored from " 0 " representing not having the symptom to " 4 " representing have the symptom all the time. The score on this scale represent " 0 " not having any of the symptoms and maximum " 28 " having all the symptoms all the time during the treatment.

"The oral symptom impact scale" has seven items that identify daily activities, (Have oral symptoms stopped you from: Having something to drink, Drinking specific beverages; Having something to eat, Eating specific food items; Have you reduced your social interactions due to oral symptoms, Stopped you form doing what you want in your daily life Reduced your quality of life).

Each item could be scored from " 0 " representing not having the symptom to " 4 " representing have the symptom all the time. The score on this scale represent " 0 " for not having any symptoms that impacted daily living and maximum " 28 " having all the symptoms that impacted all the arear measured all the time during the treatment.

The questionnaire has been face- and content validated in two steps.

In the process of content validation teen nurses and four 10 doctors working with women with breast cancer were asked to evaluate the content and give suggestion to changes if they had some. They were asked to rate the relevance of each question from 1-5. One indicated "not relevant at all" and five "very relevant". The cut off point for changing a question was set to 3.5. No questions were removed but a few changes were incorporated into the questionnaire. Next step was to present the questionnaire to ten women. They were asked to fill in the questionnaire. After filling in the questionnaire the women were interviewed about how they understood each of the questions, the rating scale, how to rate and whether all relevant options were given. Furthermore, the women were asked to evaluate the content of the questionnaire by considering if all relevant topics about oral complication were presented. Finally, the women were asked if they wanted to add questions or respond options. Hereafter the questionnaire was finalized.

Internal consistency was tested by Cronbach's Alpha. The score for "Oral symptom scale" was 0.76 and the score for "The oral symptom impact scale" was 0.83 .

\subsection{Ethics}

The women received written and oral information about the study when the questionnaire was handed out. The information stressed out that participation was voluntary, that data would be treated confidentially and that they would be anonymous in reporting of results. We only recorded the number of eligible women, who received a questionnaire and the number of questionnaires returned. If the woman did not want to participate she could just return the questionnaire in the closed envelope and it would have no influence on the present or any future treatment. No reminders were given. The study was approved but the local ethical committee and The Danish Authorities for Data Surveillance under the file no 2008-58-0028

\subsection{Statistics}

Data was processed in statistical package SPSS version 24. Descriptive statistics was used. For ration and ration interval scaled data means and SD are given. If data was normal distributed Student t-test was used to tested for differences if f-test showed non-normal distribution Mann-Whitney's rank sum test was used to test for differences between the group $p$-value level below .05 was considered as significant. A linear regression analysis was carried out using women's self-reported decrease in quality of life as the dependent variable and self-reported oral symptoms as the predictor (constant). Only results with a significant $<.10$ are reported. Pearson correlation coefficient was used to calculate correlation between sum of symptoms and changes in quality of 
life.

\section{Results}

During the study period, 101 women were referred to adjuvant CT. Their age was between 29 and 77 years with a mean age of 54.9 (8.9). All women accepted to participate and returned the questionnaires. Of the respondent $15 \%$ reported to smoke and $84 \%$ were treated with Taxotere and $16 \%$ with Taxol.

The women scored from one to 19 on the Oral symptom scale, with a mean of 8.1 (4.5). The type of oral complication and duration is presented in Table 1 . The symptoms were reported to start between day 0-9 with a mean of 3.5 (2.4) days after the initiation of the treatment. The women reported that the symptoms were most troublesome for a period of 0-35 days with a mean of 8.5 (5.5) days. Women receiving treatment with Taxotere scored 9.0 (4.3) vs 3.8 (3.2) for women treated with Taxol $(p=.000)$. Smokers scored 7.4 (4.3) vs non-smokers $8.6(4.6)$ on the Oral symptom scale $(p=.548)$.

All together 31 women reported other symptoms, such as infections $6 \%$, burning sensation in the oral cavity or tongue $4 \%$, tooth ache $2 \%$, sore lips $3 \%$. The scores on the oral symptom impact scored ranged from 0 to $20,31 \%$ of the women reported no impact on the daily living and the mean score was 3.6 (4.2). The scores from each of the items of impact on daily living are reported in Table 2. Smokers reported a score of 3.5 (4.3) vs 3.9 (4.2) for non-smokers ( $p=$ .819 , Mann-Whitney). Women treated with Taxotere scored 4.2 (4.3) vs 0.8 (2.0) for women treated with Taxol $(p=.000$, Mann-Whitney).

Table 1. Type of oral complication and duration during the adjuvant chemotherapy

\begin{tabular}{llll}
\hline Oral complication: & $\begin{array}{l}\text { Not at all } \\
\text { [CI95] }\end{array}$ & $\begin{array}{l}\text { A little or some of the time } \\
\text { [CI95] }\end{array}$ & $\begin{array}{l}\text { Most of or all the time } \\
\text { [CI95] }\end{array}$ \\
\hline \multirow{2}{*}{ Dry mouth } & $5.0 \%$ & $50.5 \%$ & $44.5 \%$ \\
& {$[1.64-11.28]$} & {$[39.83-60.17]$} & {$[34.08-54.28]$} \\
Mouth pain & $57.0 \%$ & $36.0 \%$ & $7.0 \%$ \\
& {$[456.71-66.86]$} & {$[26.64-46.21]$} & $6.3 \%-13.89]$ \\
Redness & $56.3 \%$ & $39.4 \%$ & {$[2.23-12.60]$} \\
& {$[45.72-65.92]$} & {$[29.40-49.27]$} & $20.2 \%$ \\
Coatings & $30.3 \%$ & $49.5 \%$ & {$[12.67-30.29]$} \\
& {$[21.24-39.98]$} & {$[38.86-59.20]$} & $2.0 \%$ \\
Wound & $83.5 \%$ & $14.5 \%$ & {$[0.24-7.04]$} \\
& {$[74.18-89.77]$} & {$[7.87-22.37]$} & $20.2 \%$ \\
Changes in taste & $13.3 \%$ & $36.8 \%$ & {$[12.67-29.19]$} \\
Other complications & {$[7.11-21.20]$} & {$[26.64-46.21]$} & $8.9 \%$ \\
\hline
\end{tabular}

Table 2. The impact of oral problems reported by women treated with adjuvant chemotherapy after surgery for breast cancer

\begin{tabular}{llll}
\hline Impact on daily life & $\begin{array}{l}\text { Not at all } \\
\text { [CI95] }\end{array}$ & $\begin{array}{l}\text { A little or some } \\
\text { of the time }\end{array}$ & Most of or all the time \\
\hline Have oral symptoms stopped you form having something & $81.2 \%$ & $16.4 \%$ & $2.0 \%$ \\
to drink? & {$[71.93-88.16]$} & {$[9.43-24.68]$} & {$[0.24-7.04]$} \\
Have you avoided specific beverages due to oral & $50.5 \%$ & $34.3 \%$ & $12.5 \%$ \\
symptoms? & {$[39.83-60.17]$} & {$[24.82-44.15]$} & {$[6.36-20.02]$} \\
Have oral symptoms stopped you form having something & $63.4 \%$ & $32.7 \%$ & $4.0 \%$ \\
to eat? & {$[52.76-72.44]$} & {$[23.02-42.08]$} & {$[1.10-9.93]$} \\
Have you avoided specific food items due to oral & $61.5 \%$ & $27.1 \%$ & $1.0 \%$ \\
symptoms? & {$[50.73-70.60]$} & {$[18.61-36.80]$} & {$[0.03-5.45]$} \\
Have you reduced your social interaction due to oral & $90.0 \%$ & $9 . \% 1$ & $0 \%$ \\
symptoms? & {$[82.38-95.10]$} & {$[4.20-16.40]$} & {$[0.00-3.62]$} \\
Is your quality of life been reduced due to oral symptoms? & $49.0 \%$ & $45.0 \%$ & $6.0 \%$ \\
Have oral symptoms stopped you from doing what you & {$[38.86-59.20]$} & {$[35.03-55.27]$} & {$[2.23-12.60]$} \\
want to do in your daily life? & $86.0 \%$ & $13.0 \%$ & $1.0 \%$ \\
\hline
\end{tabular}


The summarized mean symptom score was 8.1 (4.5). Women who did not report a reduction in quality of life score 5.8 (4.0) vs 11.8 (3.9) for women who reported that their quality of life was reduced most of the time $(p=.001)$. There was a positive correlation between the sum of symptoms (number of symptoms and duration) and reported quality of life score $r=.480(p=.000)$.

A linear regression analysis was carried out having the women's' score of Quality of Life as the dependent variable and the specific oral symptoms as independent variables. The results of the linear regression analysis indicated that oral symptoms were a significant predictor of reduced quality of life among women undergoing adjuvant chemotherapy ( $\mathrm{F}$ $=7.722, p$-value $\leq .000$ with an $R^{2}$ of 0.356 ) In Table 3 the result of the regression analysis is reported. Three symptoms, Coating, redness and changes of taste, accounted for $74 \%$ of the reduction in women's reported quality of life.

Table 3. Regressions analysis (Dependent variable: Reduced Quality of Life)

\begin{tabular}{lll}
\hline Model & $\boldsymbol{\beta}$ & $\boldsymbol{p}$ value \\
\hline Dry mouth & -.101 & .317 \\
Mouth pain & 0.072 & .548 \\
Rediness & 0.222 & .049 \\
Coatings & 0.271 & .013 \\
Wounds & 0.007 & .939 \\
Changes in taste & 0.256 & .018 \\
\hline
\end{tabular}

\section{Discussion}

This study is the first, to our knowledge, to describe and give an insight into how many women with breast cancer is developing oral complications during their adjuvant chemotherapy with Ebirubicin, Cyclophosphamide and Taxotere or Taxol.

All 101 women who participated in the study had some kind of oral complications as a side effect while treated with adjuvant chemotherapy. However, there was a large variation in the occurrence of the various types of oral symptoms. The regression analysis revealed that coating and changes of taste had the highest impact on women's reported reduction in quality of life due to symptoms from the oral cavity.

Previous studies have reported the OR for developing of mucositis to be approximately $4 \%$ in women receiving chemotherapy after surgery for mamma cancer. ${ }^{[8]}$ However, mucositis is an inflammatory and or ulcerative lesion of the oral tract, ${ }^{[8]}$ which only embrace a part of the oral symptoms experienced by the women. Furthermore, $95 \%$ of the women in this study reported having dry mouth for a shorter or longer period during the treatment. As saliva constitutes an important part of the mouth's immune system and thus prevents inflammation of the mouth it is important to identify and to prevent this symptom. ${ }^{[16]}$ Only identifying women with diagnosed mucositis might overlook a high number of women that could develop problems.

Taste changes are common side effects in cancer patients undergoing chemotherapy treatments. This can lead to a reduced food enjoyment and an inadequate nutrient intake with a high impact on nutritional status and quality of life. ${ }^{[17]}$

During chemotherapy for breast cancer it is reported that up to $80 \%$ of women have noticed a change in their sense of taste. ${ }^{[17,18]}$ This could contribute to reduce women's intake of food and drink and lead to a significant reduction in the intake of energy and protein causing significant reduction in BMI during the treatment. ${ }^{[15]}$ In our study approximately $50 \%$ of women reported that oral symptoms had an impact on what they chose to drink and $28 \%$ reported that their choice of food items were impacted. It is important that the nutritional state is maintained, and the staff are aware of the risk that patients do not receive adequate nutrition. ${ }^{[16]}$ As chemotherapy is given in the outpatient clinic patients could be advised to monitor their weight and discuss weight changes with the staff.

Oral symptoms in this study has been reported to range from from 2 days up to 35 days. For some of the women the oral complications were present throughout the course of treatment. The results are in line with what has been reported by others ${ }^{[15]}$ that the symptoms peak at two weeks after the onset of treatment.

In a study evaluating how quality of life is affected by oral complications, ${ }^{[19]}$ oral complications are described as the most significant side effect for chemotherapy and radiation therapy. This study shows that one of the causes of reduced quality of life due to oral complications is that these complications make it difficult to chew and sink food, gives taste changes, make it difficult to speak and causes pain. ${ }^{[19]}$ However, women with breast cancer were not included in the study. ${ }^{[19]}$ In our study $14 \%$ of the women reported that they stopped doing something in their daily lives due to the oral complications and $51 \%$ reported a reduction in quality of life. A linear regression analysis has reviled that redness, coaching and changes of taste are significant contributors to reducing quality of life and explain $74 \%$ of the reduction in the quality of life score. Being a cancer patient is difficult and challenging. Therefore, it is of great important to prevent unnecessary complications and their impact on the outcomes of the treatment, even though the complications might seem to be minor. 


\subsection{Strength and limitation}

We used a self-composed but validated questionnaire that all women who were included filled out and returned. Women reported only the symptoms from the oral cavity omitting other known side effects from chemotherapy, such as changes in smell. As women had the opportunity to report both specific and other symptoms from the oral cavity, we anticipate that we have captured the symptoms from the oral cavity women experiences, when going true chemotherapy. The collected data and the subsequent analysis of the results provide a general knowledge in relation to the research question. In order to upgrade the study further, qualitative data collection in the form of interviews of some respondents could help in a deeper understanding of the quantitative data as an interview could provide insight into the patient's perspective in relation to the sensations experienced. An interview survey based on the collected data from the questionnaire survey could help to refine and explain the quantitative data by exploring the patients' perspective in more detail. It could have given the study of oral complications a more nuanced and detailed picture of the meaning that oral complications have had for women during treatment with preventive chemotherapy.

\section{Conclusion}

Oral complications are experienced by all women who are treated with CT after breast cancer surgery. Redness, coaching and changes of taste are significant contributors to reducing quality of life. Furthermore, a significant correlation between the number and duration of symptoms was established.

\subsection{Implications for practice}

The study provides insight into which oral cavity problems the women experienced and how it affected their daily living and quality of life. These results can be used to build upon existing literature on oral cavity problems and result in providing nursing supportive care. But further studies are needed in order to get a more solid foundation for the impact and development of oral symptoms. This simple questionnaire could be used as a guide for nurses when assessing oral cavity symptoms in women having chemotherapy after breast cancer surgery.

\section{Conflicts of InTERest Disclosure}

The authors declare that there is no conflict of interest.

\section{REFERENCES}

[1] Bray F, Ferlay J, Soerjomataram I, et al. Global Cancer Statistics 2018: GLOBOCAN estimates of incidence and mortality worldwide for 36 cancers in 185 countries. CA Cancer J Clin, in press. The online GLOBOCAN 2018 database is accessible at http://gco.iarc.fr/, as part of IARC's Global Cancer Observatory.

[2] Clinical pathway for treatment of breast cancer. Danish Board of Health, 2018 [In Danish: Pakkeforløb for brystkræft]

[3] Eckhoff L, Nielsen M, Møller S, et al. TAXTOX - a retrospective study regarding the side effects of Docetaxel given as part of the adjuvant treatment to patients with primary breast cancer in Denmark from 2007 to 2009. Acta Oncologia. 2011; 50: 107582. PMid:21824038 https : //doi .org/10.3109/0284186X . 201 1.602111

[4] Jensen SB, Mouridsen HT, Bergmann OJ, et al. Oral mucosal lesions, microbial changes, and taste disturbances induced by adjuvant chemotherapy in breast cancer patients. Oral Surg Oral Med Oral Pathol Oral Radiol Endod. 2008; 106: 217-26. PMid:18554960 https://doi.org/10.1016/j.tripleo.2008.04.003

[5] Keefe D. Mucositis Management in Patients with Cancer. Supportive Cancer Therapy. 2006; 3(3): 154-57. PMid:18632489 https : //doi.org/10.3816/SCT.2006.n.013

[6] Farrington M, Cullen L, Dawson C. Evidence-Based Oral Care for Oral Mucositis. Head and Neck Nursing. 2013 Summer; 31(3): 6-15.

[7] Campos M, Aarestrup F. Oral mucositis in cancer treatment. Natural history, prevention and treatment. Mol Clin Oncol. May 2014; 2(3): 337-40.

[8] Peterson1 DE, Bensadoun RJ, Roila F. Management of oral and gastrointestinal mucositis: ESMO Clinical Practice Guidelines. Annals of Oncology. 2011; 22(Supplement 6): vi78-vi84. PMid:21908510 https://doi.org/10.1093/annonc/mdr391

[9] Borbasi S, et al. More than a sore mouth: Patients' experience of oral mucositis. Oncology Nursing Forum. 2002; 29(7): 105157. PMid:12183754 https://doi.org/10.1188/02.ONF.105 1-1057

[10] Jomar RT, Bispo VRS. The most common nursing diagnosis among adults/seniors hospitalized with cancer: intergrative review. Ecancermedicalscience. 2014; 8: 462.

[11] Available from: http://www.cancer.gov/cancertopics/pdq/ supportivecare/oralcomplications/HealthProfessiona $1 /$ page5

[12] de Vries YC, Helmich E, Karsten MDA, et al. The impact of chemosensory and food-related changes in patients with advanced oesophagogastric cancer treated with capecitabine and oxaliplatin: a qualitative study. Support Care Cancer. 2016; 24: 3119-3126. https : //doi.org/10.1007/s00520-016-3128-z

[13] Bernhardson B-M, Tishelman C, Rutqvist LE. Chemosensory Changes Experienced by Patients Undergoing Cancer Chemotherapy: A Qualitative Interview Study. J Pain Symptom Manage. 2007; 34: 403e412. PMid:17616338 https://doi.org/10.1016/j.jp ainsymman . 2006.12.010

[14] Gamper EM, Zabernigg A, Wintner LM, et al. Coming to Your Senses: Detecting Taste and Smell Alterations in Chemotherapy Patients. A Systematic Review. J Pain Symptom Manage. 2012; 44 880e895. PMid:22921177 https://doi.org/10.1016/j.jpai nsymman. 2011.11.011

[15] Boltong A, Aranda S, Keast R, et al. A Prospective Cohort Study of the Effects of Adjuvant Breast Cancer Chemotherapy on Taste Function, Food Liking, Appetite and Associated Nutritional Out- 
comes. PLOS ONE. 2014; 9(7): e103512-e103512. PMid:25078776 https://doi.org/10.1371/journal.pone.0103512

[16] Roblegg E, Coughran A, Sirjani D. Saliva: an all-rounder of our body Eur J Pharm Biopharm. 2019 Jun 17.

[17] Amézaga J, Alfaro B, Ríos Y, et al. Assessing taste and smell alterations in cancer patients undergoing chemotherapy according to treatment. Supportive Care in Cancer. 2018; 26(12): 4077-4086. PMid:29855774 https://doi.org/10.1007/s00520-018-427 $7-z$
[18] de Vries1 YC, Boesveldt S, Kelfkens CS, et al. Taste and smell perception and quality of life during and after systemic therapy for breast cancer. Breast Cancer Research and Treatment. 2018; 170: 27-34. PMid:29476290 https://doi.org/10.1007/s10549-018-472 0-3

[19] Barkokebas A, et al. Impact of oral mucositis on oral-health-related quality of life of patients diagnosed with cancer. J Oral Pathol Med. 2015; 44: 746-751. PMid:25345344 https://doi.org/10.1111/ jop. 12282 